\title{
Características físico-químicas e atividade da peroxidase e polifenoloxidase em genótipos de cupuaçu (Theobroma grandiflorum Willd ex-Spreng Schum) submetidos ao congelamento
}

\section{Physicochemical characteristes and enzymatic activity of peroxidase and polyphenoloxidase in four genotypes of cupuaçu (Theobroma grandiflorum Willd ex-Spreng Schum) submitted to freezing}

\author{
Salomão Rocha Martim ${ }^{1 *}$; José Cardoso $\mathrm{Neto}^{2}$; Ila Maria de Aguiar Oliveira²
}

\section{Resumo}

No congelamento de polpas de frutas a atividade enzimática não é completamente cessada. Podem ocorrer mudanças sensoriais, nutricionais e de coloração devido à ação de enzimas oxidativas, como a peroxidase e a polifenoloxidase. Considerando que as polpas de cupuaçu congeladas, comercializadas no Brasil, têm um prazo de validade de um ano e tornam-se escurecidas ao longo deste período, objetivou-se neste estudo avaliar o efeito do tempo de congelamento nas características físico-químicas e nas atividades da polifenoloxidase e peroxidases solúvel e insolúvel presentes nas polpas de quatro novos genótipos de cupuaçu, durante doze meses. Os frutos dos genótipos de cupuaçu, desenvolvidos pela Embrapa Amazônia Ocidental, foram despolpados, congelados e armazenados à temperatura de $-30{ }^{\circ} \mathrm{C}$. A polifenoloxidase das polpas dos quatro genótipos apresentou aumento na sua atividade com picos no sexto, nono e décimo mês e as peroxidases apresentaram oscilações na atividade enzimática. As propriedades físico-químicas das polpas apresentaram variações durante os doze meses de armazenamento sob congelamento. O teor de vitamina C dos genótipos D 28-10 e P 3-10 diminuiu a partir do $4^{\circ}$ e $10^{\circ}$ mês, respectivamente. Por outro lado os genótipos B 28-7 e P 9-8 permaneceram estáveis. Em relação à acidez em ácido cítrico, as amostras B-28-7, D 28-10 e P 9-8 não diferiram, havendo redução no genótipo $\mathrm{P}$ 3-10. O valores de $\mathrm{pH}$ e sólidos solúveis totais de todos os genótipos diminuíram ao longo do período avaliado. Houve aumento na concentração de açúcares das polpas dos genótipos B 28-7, P 3-10 e P 9-8, com exceção da amostra D 28-10 que permaneceu inalterada. Todos os genótipos apresentaram-se dentro dos padrões físico-químicos exigidos pela legislação, com exceção do genótipo P 3-10 que apresentou acidez inferior. Em relação aos parâmetros enzimáticos, houve variações na atividade das peroxidases e polifenoloxidases de todos os genótipos avaliados.

Palavras-chave: Theobroma grandiflorum, escurecimento enzimático, frutos amazônicos

\begin{abstract}
During the freezing of fruits pulps, the enzyme activity is not finished completely. Sensory, nutritional and coloring changes may occur on fruits due to the action of oxidative enzymes such as peroxidase and polyphenoloxidase. The frozen cupuaçu pulps, sold in Brazil, have a shelf life of one year and become browned during this period. The aim of this study was to evaluate the effect of frozen storage on the physicochemical characteristics, polyphenoloxidase activity and soluble and ionically bound peroxidases presented in the pulps of four new cupuaçu genotypes over twelve months. The cupuaçu
\end{abstract}

\footnotetext{
Discente de pós-graduação, Universidade Federal do Amazonas, UFAM, Manaus, AM. E-mail: salomao.martim@gmail.com Profs. Drs. UFAM, Manaus, AM. E-mail: jcardoso@ufam.edu.br; ila@ufam.edu.br Autor para correspondência
} 
genotypes developed by the West Amazonian Agroforestry Research Center (EMBRAPA) were pulped, frozen and stored at $-30{ }^{\circ} \mathrm{C}$. The polyphenoloxidase of the four cupuaçu genotypes showed an increase in activity according to the storage time with peaks in the sixth, ninth and tenth months, but the peroxidases exhibited oscillations in the enzyme activity. The physicochemical properties of the pulps showed variations during the twelve months of storage under freezing. The vitamin $\mathrm{C}$ content of D 28-10 and P 3-10 genotypes decreased from the fourth and tenth months, respectively. Moreover P 9-8 e B 28-7 genotypes remained stable. In relation the acidity of citric acid, the B-28-7, D 28-10 and P 9-8 samples were not different, but P 3-10 genotype presented a reduction. The $\mathrm{pH}$ and total soluble solids of all genotypes decreased over the study period. There was an increase in sugar concentration of B 28-7, P 3-10 and P 9-8 genotypes, except for D 28-10 sample which remained unchanged. All genotypes were in accordance with physical-chemicals standards required by legislation, except for $\mathrm{P}$ 3-10 genotype that showed a lower acidity. In respect of the enzymatic parameters, there were variations in the activity of peroxidase and polyphenoloxidases of all genotypes.

Key words: Theobroma grandiflorum, enzymatic browning, amazonian fruits

\section{Introdução}

O cupuaçuzeiro é uma arvore frutífera típica da região amazônica que figura como uma das mais promissoras dessa região, sendo crescentes os investimentos em cultivos racionais desta espécie. A importância econômica do cupuaçu está relacionada com a polpa e seus produtos derivados, como suco, licor, sorvete, geleias, doces e o cupulate, que é um produto análogo ao chocolate, obtido a partir das sementes de cupuaçu (COHEN; JACKIX, 2005).

O congelamento de polpas de frutas tornou-se uma opção viável para evitar perdas de produção, pois preserva as características originais das frutas frescas por extensos períodos e viabiliza sua comercialização nos períodos de entressafra. Além disso, alguns frutos como o cupuaçu, são bastante perecíveis, sendo seu transporte in natura por longas distâncias, praticamente inviável (MARTINS, 2008).

A comercialização de frutos na forma processada ainda enfrenta grandes desafios, pois as enzimas responsáveis pelo escurecimento de frutas e vegetais congelados não são completamente inativadas pelo frio (LOPES; MATTIETTO; MENEZES, 2005). Após o corte, ocorre o escurecimento da polpa devido à presença de compostos fenólicos e atividade das enzimas oxidativas, como a peroxidase e a polifenoloxidase (DAIUTO; VIEITES, 2008). Essas enzimas podem causar, além do escurecimento, perdas nutricionais, mudanças indesejáveis no aroma, sabor, textura e cor dos frutos, devido à ação promotora de reações de oxidação e de biodegradação em frutos e vegetais processados, ocasionando perdas econômicas (MANTOVANI; CLEMENTE, 2010).

Considerando que na literatura cientifica há carência de pesquisas em relação às enzimas oxidativas de frutos amazônicos, este estudo objetivou avaliar, por um período de um ano, as alterações físico-químicas e a atividade enzimática da peroxidase e polifenoloxidase de polpas congeladas de quatro novos genótipos de cupuaçu.

\section{Material e Métodos}

Os genótipos B 28-7, D 28-10, P 3-10, P 9-8 de cupuaçu foram coletados na área experimental da Embrapa Amazônia Ocidental, localizada na Rodovia AM-010, Km 29, zona rural de Manaus, nas coordenadas geográficas $2^{\circ} 53^{\prime} 25^{\prime}$ ' S e 59 58'06" $\mathrm{W}$ e, após lavagem dos frutos em água corrente, foram quebrados e despolpados manualmente, utilizando-se tesoura de aço inoxidável. As polpas obtidas foram fracionadas em quantidades de 50 g e acondicionadas em sacos plásticos sendo, em seguida, congeladas e armazenadas a $-30{ }^{\circ} \mathrm{C}$ em freezer com sistema digital de controle de temperatura. 
O experimento teve duração de um ano. A cada mês, foram realizadas triplicatas das análises das polpas quanto as suas características físicoquímicas e em relação às atividades enzimáticas das peroxidases solúvel e insolúvel e da polifenoloxidase.

As análises físico-químicas de $\mathrm{pH}$, acidez total, sólidos solúveis totais ( ${ }^{\circ}$ Brix) foram realizadas de acordo com as metodologias preconizadas pelo Instituto Adolfo Lutz (2008).

$\mathrm{O} \mathrm{pH}$ foi medido em potenciômetro (TECNAL, modelo Tec-2) e a acidez total por titulometria com $\mathrm{NaOH}$ 0,1M sendo os resultados expressos em porcentagem de ácido cítrico. Os sólidos solúveis totais foram medidos em refratômetro digital (QUIMIS, modelo 07678).

Os açúcares totais foram quantificados pela metodologia de Somogyi (1945) e Nelson (1944), com prévia hidrólise ácida da amostra, utilizandose espectrofotômetro UV-VIS (SHIMADZU, modelo UV-1700). Os valores foram expressos em porcentagem de glicose.

A determinação de ácido ascórbico foi realizada segundo Ranganna (1986) que se baseia na redução do ácido ascórbico pelo 2,6 diclorofenolindofenol.

As amostras de polpa dos quatro genótipos, juntamente com tampão fosfato a $0,1 \mathrm{M}, \mathrm{pH}$ 6,0, foram homogeneizadas em miniprocessador e, em seguida, centrifugadas a $4{ }^{\circ} \mathrm{C}$, sendo o sobrenadante utilizado como fonte de polifenoloxidase e de peroxidase solúvel no citoplasma celular (KHAN; ROBINSON, 1994). Para obtenção da peroxidase insolúvel, ligada covalentemente e ionicamente à parede celular, utilizou-se a metodologia descrita por Holschuh (2000) que utiliza tampão fostato 0,2 $\mathrm{M}$ pH 8,0 além de EDTA, $\mathrm{CaCl}_{2}$ e PEG nas concentrações finais de $0,01 \mathrm{M}, 0,2 \mathrm{M}$ e $2 \%$, respectivamente.

A atividade da polifenoloxidase foi determinada usando-se catecol como substrato, conforme descrito por Oktay et al. (1995). Já as atividades das peroxidases solúvel e insolúvel foram determinadas usando-se guaiacol e peróxido de hidrogênio como substratos, conforme descrito por Khan e Robinson (1994). Para todas as análises enzimáticas, uma unidade de atividade enzimática foi definida como a quantidade de enzima que causou um aumento de 0,001 unidades de absorbância por minuto/g de amostra.

\section{Delineamento estatístico}

Inicialmente foi realizada uma análise descritiva de cada uma das variáveis: peroxidase solúvel, peroxidase insolúvel, polifenoloxidase, ácido ascórbico, ácido cítrico, açúcares totais, pH, sólidos solúveis totais .

O delineamento experimental utilizado, inicialmente, para todas as variáveis, foi um fatorial 4x12 com três replicações verdadeiras. Apenas a variável "sólidos solúveis totais" satisfez as pressuposições de normalidade e homocedasticidade. Para as outras variáveis foi utilizado teste de Kruskal-Wallis como alternativa para a análise de variância. As comparações múltiplas, quando necessárias, foram realizadas através do teste de Tukey. Todos os testes foram realizados usando nível de 5\% de significância.

\section{Resultados e Discussão}

A Instrução Normativa $N^{\circ} 1$ de 07 de janeiro de 2000, do Ministério da Agricultura, Pecuária e Abastecimento (MAPA) recomenda os seguintes padrões mínimos de qualidade para as polpas de cupuaçu: ácido ascórbico (18,00 mg/100 g), $\mathrm{pH}$ $(2,60)$, acidez expressa em ácido cítrico $(1,50$ $\mathrm{g} / 100 \mathrm{~g})$, açúcares totais $(6,00 \mathrm{~g} / 100 \mathrm{~g})$ e sólidos solúveis em ${ }^{\circ}$ Brix $(9,00)$ (BRASIL, 2000) .Os dados referentes à composição físico-química das polpas dos genótipos B 28-7, D 28-10, P 3-10 e P 9-8 encontram-se nas tabelas 1, 2, 3 e 4, respectivamente. 
Tabela 1. Efeito do tempo de congelamento na composição físico-química da polpa de cupuaçu- Genótipo B 28-7.

\begin{tabular}{|c|c|c|c|c|c|}
\hline Tempo & $\begin{array}{l}\text { Açúcar total } \\
(\mathrm{g} / 100 \mathrm{~g})^{* *}\end{array}$ & $\begin{array}{l}\text { Ácido ascórbico } \\
(\mathrm{mg} / 100 \mathrm{~g})^{* *}\end{array}$ & $\begin{array}{l}\text { Acidez em ácido } \\
\text { cítrico }(\mathrm{g} / 100 \mathrm{~g})^{* *}\end{array}$ & $\mathrm{pH}^{* *}$ & $\begin{array}{l}\text { Sólidos solúveis } \\
\left({ }^{\circ} \text { Brix }\right) *\end{array}$ \\
\hline 0 & $7,45 \pm 0,02^{\mathrm{f}}$ & $27,50 \pm 0,00^{\mathrm{a}}$ & $1,51 \pm 0,00^{\mathrm{a}}$ & $3,37 \pm 0,01^{\mathrm{a}}$ & $13,40 \pm 0,00^{\mathrm{a}}$ \\
\hline 1 & $7,45 \pm 0,02^{\mathrm{f}}$ & $27,50 \pm 0,00^{\mathrm{a}}$ & $1,51 \pm 0,00^{\mathrm{a}}$ & $3,37 \pm 0,01^{\mathrm{a}}$ & $13,40 \pm 0,00^{\mathrm{a}}$ \\
\hline 2 & $7,95 \pm 0,03^{\mathrm{e}}$ & $27,49 \pm 0,01^{\mathrm{b}}$ & $1,51 \pm 0,01^{\mathrm{ab}}$ & $3,36 \pm 0,00^{\mathrm{b}}$ & $13,13 \pm 0,11^{\mathrm{b}}$ \\
\hline 3 & $7,95 \pm 0,03^{\mathrm{e}}$ & $27,49 \pm 0,01^{\mathrm{b}}$ & $1,51 \pm 0,01^{\mathrm{ab}}$ & $3,36 \pm 0,00^{\mathrm{b}}$ & $13,13 \pm 0,11^{b}$ \\
\hline 4 & $8,75 \pm 0,03^{\mathrm{d}}$ & $27,46 \pm 0,01^{\mathrm{c}}$ & $1,50 \pm 0,00^{\mathrm{bc}}$ & $3,36 \pm 0,01^{\mathrm{b}}$ & $13,00 \pm 0,00^{\mathrm{b}}$ \\
\hline 5 & $8,75 \pm 0,03^{\mathrm{d}}$ & $27,46 \pm 0,01^{\mathrm{c}}$ & $1,50 \pm 0,00^{\mathrm{bc}}$ & $3,36 \pm 0,01^{\mathrm{b}}$ & $13,00 \pm 0,00^{\mathrm{b}}$ \\
\hline 6 & $8,93 \pm 0,02^{\mathrm{c}}$ & $27,42 \pm 0,00^{\mathrm{d}}$ & $1,50 \pm 0,01^{\mathrm{cd}}$ & $3,31 \pm 0,01^{\mathrm{c}}$ & $12,47 \pm 0,06^{\mathrm{b}}$ \\
\hline 7 & $8,93 \pm 0,02^{c}$ & $27,42 \pm 0,00^{\mathrm{d}}$ & $1,50 \pm 0,01^{\mathrm{cd}}$ & $3,31 \pm 0,01^{\mathrm{c}}$ & $12,47 \pm 0,06^{\mathrm{b}}$ \\
\hline 8 & $8,97 \pm 0,02^{\mathrm{b}}$ & $27,41 \pm 0,01^{\mathrm{e}}$ & $1,49 \pm 0,01^{\mathrm{de}}$ & $3,28 \pm 0,01^{\mathrm{d}}$ & $12,40 \pm 0,10^{\mathrm{b}}$ \\
\hline 9 & $8,97 \pm 0,02^{b}$ & $27,41 \pm 0,01^{\mathrm{e}}$ & $1,49 \pm 0,01^{\mathrm{de}}$ & $3,28 \pm 0,01^{\mathrm{d}}$ & $12,40 \pm 0,10^{\mathrm{b}}$ \\
\hline 10 & $9,13 \pm 0,15^{\mathrm{a}}$ & $27,38 \pm 0,01^{\mathrm{f}}$ & $1,49 \pm 0,00^{\mathrm{e}}$ & $3,26 \pm 0,01^{\mathrm{e}}$ & $12,33 \pm 0,15^{\mathrm{b}}$ \\
\hline 11 & $9,13 \pm 0,15^{\mathrm{a}}$ & $27,38 \pm 0,01^{\mathrm{f}}$ & $1,49 \pm 0,00^{\mathrm{e}}$ & $3,26 \pm 0,01^{\mathrm{e}}$ & $12,33 \pm 0,15^{\mathrm{b}}$ \\
\hline 12 & $9,30 \pm 0,03^{\mathrm{a}}$ & $27,37 \pm 0,01^{\mathrm{f}}$ & $1,49 \pm 0,00^{\mathrm{e}}$ & $3,20 \pm 0,01^{\mathrm{e}}$ & $12,43 \pm 0,06^{\mathrm{b}}$ \\
\hline p-valor & 0,000230675 & 0,000221121 & 0,001696295 & 0,000298237 & $<0,00001$ \\
\hline
\end{tabular}

Dados expressos como média de triplicata \pm desvio-padrão. Médias seguidas da mesma letra, nas colunas, não diferem estatisticamente entre si $(\mathrm{p}>0,05)$. Testes submetidos ao Teste de Tukey*. Testes submetidos ao Teste de Kruskal-Wallis **.

Fonte: Elaboração dos autores.

Tabela 2. Efeito do tempo de congelamento na composição físico-química da polpa de cupuaçu-Genótipo D 28-10.

\begin{tabular}{cccccc}
\hline Tempo & $\begin{array}{c}\text { Açúcar total } \\
(\mathrm{g} / 100 \mathrm{~g})^{* *}\end{array}$ & $\begin{array}{c}\text { Ácido ascórbico } \\
(\mathrm{mg} / 100 \mathrm{~g})^{* *}\end{array}$ & $\begin{array}{c}\text { Acidez em ácido } \\
\text { cítrico } \\
(\mathrm{g} / 100 \mathrm{~g})^{* *}\end{array}$ & $\mathrm{pH}^{* *}$ & $\begin{array}{c}\text { Sólidos solúveis } \\
\left({ }^{\circ} \text { Brix }\right)^{*}\end{array}$ \\
\hline 0 & $10,75 \pm 0,10^{\mathrm{abc}}$ & $19,73 \pm 0,06^{\mathrm{a}}$ & $1,94 \pm 0,01^{\mathrm{a}}$ & $4,00 \pm 0,01^{\mathrm{a}}$ & $16,62 \pm 0,01^{\mathrm{a}}$ \\
1 & $10,75 \pm 0,10^{\mathrm{abc}}$ & $19,73 \pm 0,06^{\mathrm{a}}$ & $1,94 \pm 0,01^{\mathrm{a}}$ & $4,00 \pm 0,01^{\mathrm{a}}$ & $16,62 \pm 0,01^{\mathrm{a}}$ \\
2 & $7,76 \pm 5,17^{\mathrm{e}}$ & $19,60 \pm 0,26^{\mathrm{a}}$ & $1,94 \pm 0,00^{\mathrm{ab}}$ & $3,99 \pm 0,00^{\mathrm{a}}$ & $16,61 \pm 0,01^{\mathrm{a}}$ \\
3 & $7,76 \pm 5,17^{\mathrm{e}}$ & $19,60 \pm 0,26^{\mathrm{a}}$ & $1,94 \pm 0,00^{\mathrm{ab}}$ & $3,99 \pm 0,00^{\mathrm{a}}$ & $16,61 \pm 0,01^{\mathrm{a}}$ \\
4 & $10,76 \pm 0,00^{\mathrm{d}}$ & $19,00 \pm 0,00^{\mathrm{b}}$ & $1,94 \pm 0,01^{\mathrm{abc}}$ & $3,97 \pm 0,00^{\mathrm{b}}$ & $16,27 \pm 0,11^{\mathrm{b}}$ \\
5 & $10,76 \pm 0,00^{\mathrm{d}}$ & $19,00 \pm 0,00^{\mathrm{b}}$ & $1,94 \pm 0,01^{\mathrm{abc}}$ & $3,97 \pm 0,00^{\mathrm{b}}$ & $16,27 \pm 0,11^{\mathrm{b}}$ \\
6 & $10,73 \pm 0,06^{\mathrm{c}}$ & $19,00 \pm 0,00^{\mathrm{b}}$ & $1,93 \pm 0,01^{\mathrm{bcd}}$ & $3,94 \pm 0,01^{\mathrm{c}}$ & $15,73 \pm 0,11^{\mathrm{b}}$ \\
7 & $10,73 \pm 0,06^{\mathrm{c}}$ & $19,00 \pm 0,00^{\mathrm{b}}$ & $1,93 \pm 0,01^{\mathrm{bcd}}$ & $3,94 \pm 0,01^{\mathrm{c}}$ & $15,73 \pm 0,11^{\mathrm{b}}$ \\
8 & $10,78 \pm 0,01^{\mathrm{b}}$ & $18,93 \pm 0,06^{\mathrm{bc}}$ & $1,93 \pm 0,00^{\mathrm{cd}}$ & $3,91 \pm 0,01^{\mathrm{d}}$ & $15,50 \pm 0,00^{\mathrm{b}}$ \\
9 & $10,78 \pm 0,01^{\mathrm{b}}$ & $18,93 \pm 0,06^{\mathrm{bc}}$ & $1,93 \pm 0,00^{\mathrm{cd}}$ & $3,91 \pm 0,01^{\mathrm{d}}$ & $15,50 \pm 0,00^{\mathrm{b}}$ \\
10 & $10,78 \pm 0,00^{\mathrm{a}}$ & $18,90 \pm 0,10^{\mathrm{c}}$ & $1,93 \pm 0,01^{\mathrm{d}}$ & $3,89 \pm 0,01^{\mathrm{d}}$ & $15,37 \pm 0,11^{\mathrm{b}}$ \\
11 & $10,78 \pm 0,00^{\mathrm{a}}$ & $18,90 \pm 0,10^{\mathrm{c}}$ & $1,93 \pm 0,01^{\mathrm{d}}$ & $3,89 \pm 0,01^{\mathrm{d}}$ & $15,37 \pm 0,11$ \\
12 & $10,80 \pm 0,02^{\mathrm{a}}$ & $18,80 \pm 0,10^{\mathrm{c}}$ & $1,92 \pm 0,01^{\mathrm{cd}}$ & $3,96 \pm 0,01^{\mathrm{e}}$ & $15,18 \pm 0,15^{\mathrm{b}}$ \\
$\mathrm{p}$-valor & 0,02700834 & 0,001004204 & 0,01577069 & $0,0002431^{2}$ & $<0,00001$ \\
\hline
\end{tabular}

Dados expressos como média de triplicata \pm desvio-padrão. Médias seguidas da mesma letra, nas colunas, não diferem estatisticamente entre si $(\mathrm{p}>0,05)$. Testes submetidos ao Teste de Tukey *. Testes submetidos ao Teste de Kruskal-Wallis $* *$.

Fonte: Elaboração dos autores. 
Tabela 3. Efeito do tempo de congelamento na composição físico - química da polpa de cupuaçu - Genótipo P 3. 10.

\begin{tabular}{cccccc}
\hline Tempo & $\begin{array}{c}\text { Açúcar total } \\
(\mathrm{g} / 100 \mathrm{~g})^{* *}\end{array}$ & $\begin{array}{c}\text { Ácido ascórbico } \\
(\mathrm{mg} / 100 \mathrm{~g})^{* *}\end{array}$ & $\begin{array}{c}\text { Acidez em ácido } \\
\text { ć́trico } \\
(\mathrm{g} / 100 \mathrm{~g})^{* *}\end{array}$ & $\mathrm{pH}^{* *}$ & $\begin{array}{c}\text { Sólidos solúveis } \\
\left({ }^{\circ} \text { Brix }\right)^{*}\end{array}$ \\
\hline 0 & $12,76 \pm 0,01^{\mathrm{d}}$ & $28,07 \pm 0,06^{\mathrm{a}}$ & $0,76 \pm 0,01^{\mathrm{a}}$ & $3,62 \pm 0,00^{\mathrm{a}}$ & $15,00 \pm 0,00^{\mathrm{a}}$ \\
1 & $12,76 \pm 0,01^{\mathrm{d}}$ & $28,07 \pm 0,06^{\mathrm{a}}$ & $0,76 \pm 0,01^{\mathrm{a}}$ & $3,62 \pm 0,00^{\mathrm{a}}$ & $15,00 \pm 0,00^{\mathrm{a}}$ \\
2 & $12,77 \pm 0,01^{\mathrm{d}}$ & $28,03 \pm 0,06^{\mathrm{ab}}$ & $0,75 \pm 0,00^{\mathrm{b}}$ & $3,62 \pm 0,01^{\mathrm{a}}$ & $14,90 \pm 0,00^{\mathrm{a}}$ \\
3 & $12,77 \pm 0,01^{\mathrm{d}}$ & $28,03 \pm 0,06^{\mathrm{ab}}$ & $0,75 \pm 0,00^{\mathrm{b}}$ & $3,62 \pm 0,01^{\mathrm{a}}$ & $14,90 \pm 0,00^{\mathrm{a}}$ \\
4 & $12,84 \pm 0,02^{\mathrm{c}}$ & $28,00 \pm 0,00^{\mathrm{b}}$ & $0,74 \pm 0,01^{\mathrm{c}}$ & $3,62 \pm 0,01^{\mathrm{a}}$ & $14,90 \pm 0,01^{\mathrm{a}}$ \\
5 & $12,84 \pm 0,02^{\mathrm{c}}$ & $28,00 \pm 0,00^{\mathrm{b}}$ & $0,74 \pm 0,01^{\mathrm{c}}$ & $3,62 \pm 0,01^{\mathrm{a}}$ & $14,90 \pm 0,0^{\mathrm{a}} 1$ \\
6 & $12,84 \pm 0,02^{\mathrm{c}}$ & $27,98 \pm 0,01^{\mathrm{c}}$ & $0,60 \pm 0,00^{\mathrm{d}}$ & $3,61 \pm 0,01^{\mathrm{b}}$ & $14,88 \pm 0,02^{\mathrm{a}}$ \\
7 & $12,84 \pm 0,02^{\mathrm{c}}$ & $27,98 \pm 0,01^{\mathrm{c}}$ & $0,60 \pm 0,00^{\mathrm{d}}$ & $3,61 \pm 0,01^{\mathrm{b}}$ & $14,88 \pm 0,02^{\mathrm{a}}$ \\
8 & $13,00 \pm 0,00^{\mathrm{b}}$ & $27,96 \pm 0,01^{\mathrm{c}}$ & $0,60 \pm 0,00^{\mathrm{d}}$ & $3,59 \pm 0,01^{\mathrm{c}}$ & $14,71 \pm 0,01^{\mathrm{b}}$ \\
9 & $13,00 \pm 0,00^{\mathrm{b}}$ & $27,96 \pm 0,01^{\mathrm{c}}$ & $0,60 \pm 0,00^{\mathrm{d}}$ & $3,59 \pm 0,01^{\mathrm{c}}$ & $14,71 \pm 0,01^{\mathrm{b}}$ \\
10 & $13,11 \pm 0,01^{\mathrm{a}}$ & $27,78 \pm 0,00^{\mathrm{d}}$ & $0,60 \pm 0,01^{\mathrm{d}}$ & $3,58 \pm 0,00^{\mathrm{c}}$ & $14,51 \pm 0,01^{\mathrm{b}}$ \\
11 & $13,11 \pm 0,01^{\mathrm{a}}$ & $27,78 \pm 0,00^{\mathrm{d}}$ & $0,60 \pm 0,01^{\mathrm{d}}$ & $3,58 \pm 0,00^{\mathrm{c}}$ & $14,51 \pm 0,01^{\mathrm{b}}$ \\
12 & $13,12 \pm 0,01^{\mathrm{a}}$ & $27,78 \pm 0,01^{\mathrm{d}}$ & $0,59 \pm 0,00^{\mathrm{e}}$ & $3,58 \pm 0,00^{\mathrm{c}}$ & $14,13 \pm 0,01^{\mathrm{b}}$ \\
$\mathrm{p}$-valor & 0,000331806 & 0,00035149 & 0,0002617 & 0,000488424 & $<0,00001$ \\
\hline
\end{tabular}

Dados expressos como média de triplicata \pm desvio-padrão. Médias seguidas da mesma letra, nas colunas, não diferem estatisticamente entre si $(\mathrm{p}>0,05)$. Testes submetidos ao Teste de Tukey*. Testes submetidos ao Teste de Kruskal-Wallis **.

Fonte: Elaboração dos autores.

Tabela 4. Efeito do tempo de congelamento na composição físico-química da polpa de cupuaçu- Genótipo P 9-8.

\begin{tabular}{cccccc}
\hline Tempo & $\begin{array}{c}\text { Açúcar total } \\
(\mathrm{g} / 100 \mathrm{~g})^{* *}\end{array}$ & $\begin{array}{c}\text { Ácido ascórbico } \\
(\mathrm{mg} / 100 \mathrm{~g})^{* *}\end{array}$ & $\begin{array}{c}\text { Acidez em ácido } \\
\text { ć́trico } \\
(\mathrm{g} / 100 \mathrm{~g}))^{* *}\end{array}$ & $\mathrm{pH}^{* *}$ & $\begin{array}{c}\text { Sólidos solúveis } \\
\left({ }^{\circ} B \operatorname{Brix}\right)^{*}\end{array}$ \\
\hline 0 & $8,12 \pm 0,02^{\mathrm{g}}$ & $24,39 \pm 0,01^{\mathrm{a}}$ & $1,47 \pm 0,00^{\mathrm{a}}$ & $3,77 \pm 0,01^{\mathrm{a}}$ & $12,00 \pm 0,00^{\mathrm{a}}$ \\
1 & $8,12 \pm 0,02^{\mathrm{g}}$ & $24,39 \pm 0,01^{\mathrm{a}}$ & $1,47 \pm 0,00^{\mathrm{a}}$ & $3,77 \pm 0,01^{\mathrm{a}}$ & $12,00 \pm 0,00^{\mathrm{a}}$ \\
2 & $8,16 \pm 0,01^{\mathrm{f}}$ & $24,38 \pm 0,01^{\mathrm{ab}}$ & $1,47 \pm 0,01^{\mathrm{a}}$ & $3,75 \pm 0,00^{\mathrm{b}}$ & $12,00 \pm 0,00^{\mathrm{a}}$ \\
3 & $8,16 \pm 0,01^{\mathrm{f}}$ & $24,38 \pm 0,01^{\mathrm{ab}}$ & $1,47 \pm 0,01^{\mathrm{a}}$ & $3,75 \pm 0,00^{\mathrm{b}}$ & $12,00 \pm 0,00^{\mathrm{a}}$ \\
4 & $8,29 \pm 0,01^{\mathrm{e}}$ & $24,37 \pm 0,00^{\mathrm{bc}}$ & $1,46 \pm 0,01^{\mathrm{a}}$ & $3,75 \pm 0,01^{\mathrm{b}}$ & $11,00 \pm 0,00^{\mathrm{b}}$ \\
5 & $8,29 \pm 0,01^{\mathrm{e}}$ & $24,37 \pm 0,00^{\mathrm{bc}}$ & $1,46 \pm 0,01^{\mathrm{a}}$ & $3,75 \pm 0,01^{\mathrm{b}}$ & $11,00 \pm 0,00^{\mathrm{b}}$ \\
6 & $8,39 \pm 0,01^{\mathrm{d}}$ & $24,36 \pm 0,01^{\mathrm{cd}}$ & $1,46 \pm 0,00^{\mathrm{a}}$ & $3,74 \pm 0,00^{\mathrm{c}}$ & $10,87 \pm 0,11^{\mathrm{b}}$ \\
7 & $8,39 \pm 0,01^{\mathrm{d}}$ & $24,36 \pm 0,01^{\mathrm{cd}}$ & $1,46 \pm 0,00^{\mathrm{a}}$ & $3,74 \pm 0,00^{\mathrm{c}}$ & $10,87 \pm 0,11^{\mathrm{b}}$ \\
8 & $8,46 \pm 0,01^{\mathrm{c}}$ & $24,35 \pm 0,01^{\mathrm{d}}$ & $1,46 \pm 0,01^{\mathrm{a}}$ & $3,72 \pm 0,01^{\mathrm{d}}$ & $10,77 \pm 0,06^{\mathrm{b}}$ \\
9 & $8,46 \pm 0,01^{\mathrm{c}}$ & $24,35 \pm 0,01^{\mathrm{d}}$ & $1,46 \pm 0,01^{\mathrm{a}}$ & $3,72 \pm 0,01^{\mathrm{d}}$ & $10,77 \pm 0,06^{\mathrm{b}}$ \\
10 & $8,57 \pm 0,01^{\mathrm{b}}$ & $24,35 \pm 0,01^{\mathrm{d}}$ & $1,46 \pm 0,00^{\mathrm{a}}$ & $3,71 \pm 0,01^{\mathrm{d}}$ & $10,43 \pm 0,06^{\mathrm{b}}$ \\
11 & $8,57 \pm 0,01^{\mathrm{b}}$ & $24,35 \pm 0,01^{\mathrm{d}}$ & $1,46 \pm 0,00^{\mathrm{a}}$ & $3,71 \pm 0,01^{\mathrm{d}}$ & $10,43 \pm 0,06^{\mathrm{b}}$ \\
12 & $8,63 \pm 0,02^{\mathrm{a}}$ & $24,36 \pm 0,30^{\text {cd }}$ & $1,46 \pm 0,01^{\mathrm{a}}$ & $3,70 \pm 0,01^{\mathrm{d}}$ & $10,40 \pm 0,00^{\mathrm{b}}$ \\
p-valor & 0,000199311 & 0,00159547 & 0,2190273 & 0,000376684 & $<0,00001$ \\
\hline
\end{tabular}

Dados expressos como média de triplicata \pm desvio-padrão. Médias seguidas da mesma letra, nas colunas, não diferem estatisticamente entre si ( $>$ > 0,05). Testes submetidos ao Teste de Tukey*. Testes submetidos ao Teste de Kruskal-Wallis **. Fonte: Elaboração dos autores. 
$\mathrm{Na}$ análise do conteúdo de vitamina $\mathrm{C}$ dos genótipos, durante o período de 12 meses de congelamento, a amostra P 3-10 foi a que apresentou maior teor de acido ascórbico para a polpa in natura (28,06 mg / $100 \mathrm{~g})$, no tempo zero, e após um ano de congelamento $(27,77 \mathrm{mg} / 100 \mathrm{~g})$. Esses valores estão de acordo com as recomendações do MAPA e são superiores ao resultado de 5,05 mg/100 g de ácido ascórbico encontrado por Santos et al. (2010) para a polpa de cupuaçu. A análise estatística das médias dos resultados apresentados nas tabelas 1 , 2,3 e 4 demonstrou que o conteúdo de vitamina $\mathrm{C}$ dos genótipos D 28-10 e P 3-10 diminuiu, a partir do $4^{\circ}$ e $10^{\circ}$ mês de congelamento, respectivamente. Parâmetros físico-químicos, como os teores de vitamina $C$, são afetados não somente pelas características genéticas das plantas, mas, também, por outros fatores como temperatura, precipitações fluviais e tipo de adubação (NOGUEIRA et al., 2002). O congelamento não afetou o conteúdo de vitamina C das amostras B 28-7 e P 9-8, corroborando com os resultados obtidos por Aquino, Moés e Castro (2011) que também não constataram a influência do congelamento em frutos de acerola congelados por diferentes métodos criogênicos.

Quanto ao $\mathrm{pH}$ das polpas in natura dos quatro genótipos, verificou-se que os valores médios variaram de 3,37 a 4,00. Esses valores foram similares aos reportados por Porte et al. (2010) e Costa (2002) que encontraram $\mathrm{pH}$ de 3,60 e 3,34, respectivamente. Todas as polpas avaliadas estavam de acordo com a legislação vigente que estipula um valor mínimo de pH 2,6 para polpas de cupuaçu (BRASIL, 2000). A análise estatística detectou que o pH de todos os genótipos diminuiu, durante os 12 meses de congelamento.

Em relação à acidez em ácido cítrico, as polpas in natura dos genótipos B 28-7 e D 28-10 apresentaram, respectivamente, valores de 1,51 e 1,94 g/100 g, estando, portanto, dentro dos padrões de qualidade exigidos pelo MAPA que estipula um teor mínimo de $1,5 \mathrm{~g} / 100 \mathrm{~g}$ e similares aos valores de $2,17 \mathrm{~g} / 100$ $\mathrm{g}$ e $2,70 \mathrm{~g} / 100 \mathrm{~g}$ observados, respectivamente, por
Oliveira (2006) e Araújo (2007). Após um ano de congelamento essas amostras não diferiram estatisticamente. Observou-se um teor de acidez $1,47 \mathrm{~g} / 100 \mathrm{~g}$ para a amostra in natura do genótipo P 9-8 que permaneceu estável durante o seu armazenamento a temperatura de $-30{ }^{\circ} \mathrm{C}$, porém a polpa in natura do genótipo P 3-10 apresentou uma acidez de 0,76 g/100 g e, após 12 meses de congelamento, houve uma redução para $0,59 \mathrm{~g} / 100$ g. Freire et al. (2009) analisaram polpas de cupuaçu com uma semana de congelamento e constataram uma acidez expressa em ácido cítrico entre 1,38 e $1,87 \mathrm{~g} / 100 \mathrm{~g}$. Esses teores foram inferiores ao das polpas de três genótipos de cupuaçu com um ano de congelamento que variaram de 1,49 a 1,92 g/100 g, com exceção do genótipo P 3-10 que apresentou uma acidez de 0,59 g/100 g.

Alterações no teor de acidez total e pH ocorrem devido a mudanças nos conteúdos de ácidos orgânicos nos produtos (MULYAWANTI; DEWANDARI; YULIANINGSIH, 2010). De acordo com Sahari, Mohsen e Zohreh (2004) essas modificações podem ser influenciadas pelo tempo de armazenamento, reações enzimáticas, método de congelamento e pela presença de microrganismos.

A análise estatística detectou um aumento em função do tempo de congelamento na concentração de açúcares das polpas dos genótipos B 28-7, P 3-10 e P 9-8, com exceção do D 28-10 que permaneceu estável. A polpa in natura do genótipo P 3-10 apresentou a maior concentração de açúcares totais $(12,76$ g/ 100 g). Oliveira (2006) e Araújo (2007) detectaram, respectivamente, teores de 9,12 g/ 100 $\mathrm{g}$ e $8,60 \mathrm{~g} / 100 \mathrm{~g}$ de açúcares totais em polpas de cupuaçu, valores inferiores aos encontrados neste estudo. A elevação nas concentrações de açúcares totais também foi observada por Evangelista e Vieites (2006), em polpas de goiaba congeladas e armazenadas a $-18{ }^{\circ} \mathrm{C}$. Lopes, Mattietto e Menezes (2005) verificaram discreta elevação dos teores de açúcares totais ao avaliar a estabilidade da polpa de pitanga submetida ao congelamento e armazenada por 90 dias. Silva et al. (2010), ao estudarem 
a estabilidade da polpa de bacuri, verificaram diferenças nos teores de açúcares totais nas amostras analisadas. Os açúcares totais variaram de 10,11 a $13,65 \%$ nas polpas de bacuri armazenadas a $-20{ }^{\circ} \mathrm{C}$, durante doze meses. Segundo Chitarra e Chitarra (2005), a hidrólise do amido, a degradação de polissacarídeos das paredes celulares e a perda de água pelos frutos podem contribuir para o aumento nos conteúdo de açúcares durante o período de armazenamento.

O maior valor de sólidos solúveis totais foi encontrado na polpa in natura do genótipo D 28$10(16,62 \%)$ e o menor na amostra P 9-8 (12,00 $\%$ ), estando de acordo com a legislação vigente. No decorrer do período de um ano de armazenamento das polpas congeladas dos quatro genótipos de cupuaçu, houve uma redução no conteúdo de sólidos solúveis totais. Brunini, Oliveira e Varanda (2003) também verificaram uma diminuição nos teores de sólidos solúveis totais ao avaliar a qualidade de polpa de goiaba "paluma" armazenada a $-20{ }^{\circ} \mathrm{C}$, durante 24 semanas. Segundo Faraoni (2006) o decréscimo dos teores de sólidos solúveis totais pode ocorrer devido à formação de grandes cristais de gelo decorrente do congelamento lento. Yamashita et al. (2003) observaram diminuição dos teores de sólidos solúveis ao avaliar produtos obtidos a partir da acerola e atribuiu esse decréscimo à atividade enzimática, mesmo estando os produtos em temperaturas de congelamento.

De acordo com o teste não paramétrico de Kruskal-Wallis houve diferença entre os genótipos para as atividades enzimáticas das peroxidase solúvel, insolúvel e da polifenoloxidase.

Nas análises realizadas com a polpa in natura, (t0), o genótipo que apresentou maior atividade enzimática de peroxidase insolúvel foi a P 9-8 com 45.833,33 U/g/min, seguido do D 28-10 (39.333,33 $\mathrm{U} / \mathrm{g} / \mathrm{min})$, B $28-7$ (34.166,66 U/g/min) e P 3-10 (24.000 U/g/min), conforme figura 1. Porém, após um mês de congelamento houve acentuado declínio da atividade enzimática em todos os genótipos. Nos meses seguintes houve oscilações de atividade enzimática das amostras B 28-7, D 28-10 e P 3-10 ao contrário do genótipo P 9-8 que apresentou tendente decréscimo de atividade. Berbicz e Clemente (2001) determinaram a peroxidase insolúvel extraída da polpa de laranja e constataram valores de até 11,45 U/g/min. Neves (2002) relatou atividade enzimática de $10.000 \mathrm{U} / \mathrm{g} / \mathrm{min}$ para a peroxidase insolúvel extraída da polpa de pêssego. Freitas et al. (2008) observaram valores de 2,97 e $2,83 \mathrm{U} / \mathrm{g} / \mathrm{min}$ de atividade de peroxidase insolúvel para uvas das variedades Benitaka e Rubi, respectivamente.

Figura 1. Atividade da peroxidase insolúvel nos genótipos de cupuaçu armazenados sob congelamento.

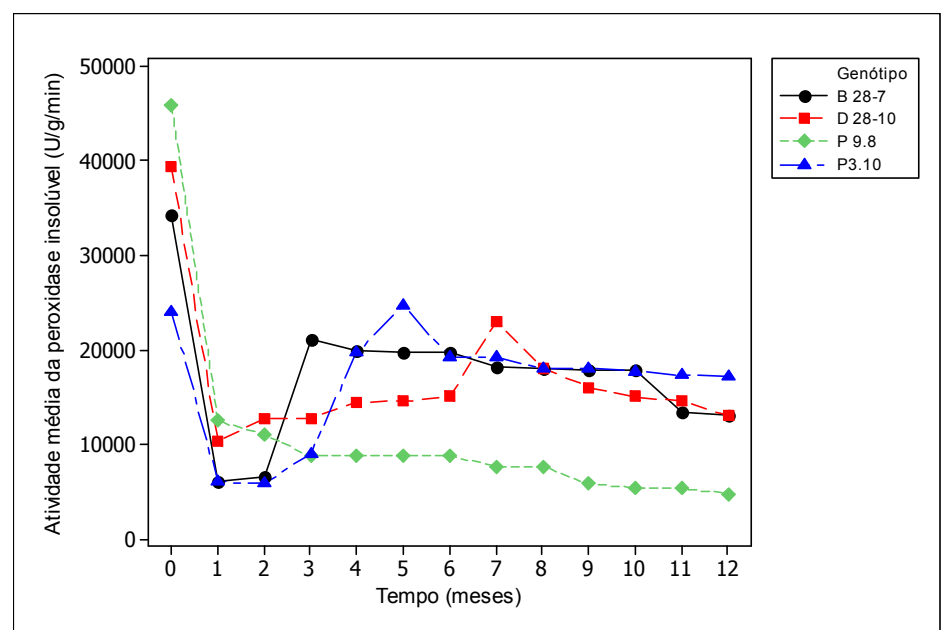

Fonte: Elaboração dos autores. 
Em relação à peroxidase solúvel, o genótipo D 28-10 foi o que apresentou maior atividade (14.722,22 U/g/min), conforme demonstra a figura 2. Foi observado um declínio na atividade enzimática das peroxidases solúvel e insolúvel dos quatro genótipos de cupuaçu, após um mês de congelamento. Houve oscilações na atividade dessas enzimas nos meses seguintes, sendo que no décimo mês de armazenamento todas as amostras apresentaram picos na atividade da peroxidase solúvel. O genótipo $\mathrm{P}$ 9-8 foi o que apresentou menor atividade de peroxidase solúvel (1.000 $\mathrm{U} / \mathrm{g} / \mathrm{min}$ ), o que foi observado após um mês do congelamento inicial. Ferreira (2009) ao analisar a atividade da peroxidase solúvel de polpas de cupuaçu congeladas, procedentes do estado de Roraima e município de Itacoatiara - AM, constatou que a atividade inicial foi de 16.780 $\mathrm{U} / \mathrm{g} / \mathrm{min}$ e $18.067 \mathrm{U} / \mathrm{g} / \mathrm{min}$, respectivamente, com uma redução de $52 \%$ e $55 \%$, após dois meses de armazenamento sob congelamento, com oscilações durante os doze meses de estocagem. Nunes et al. (2010) observaram aumento da atividade enzimática da peroxidase ao analisar o efeito de diferentes temperaturas na qualidade da mandioquinha minimamente processada e armazenada a $0{ }^{\circ} \mathrm{C}$ durante 15 dias. Segundo Cano, Marin e Fúster (1990) a solubilidade da peroxidase pode ser afetada pela perda da integridade da membrana celular como resultado do crescimento dos cristais de gelo no tecido promovendo o incremento da atividade residual dessa enzima.

Figura 2. Atividade da peroxidase solúvel nos genótipos de cupuaçu armazenados sob congelamento.

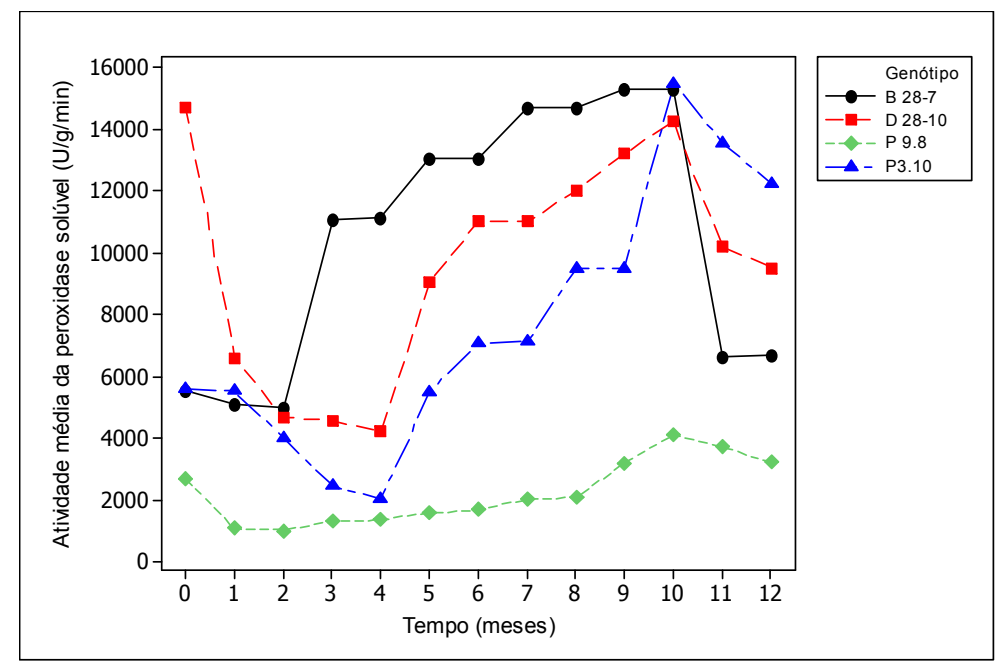

Fonte: Elaboração dos autores.

Em relação à polifenoloxidase, observou-se elevação da atividade em todos os genótipos, tendo a amostra P 9-8 apresentado maior atividade (140 $\mathrm{U} / \mathrm{g} / \mathrm{min}$ ) com 9 meses de congelamento e a B 28-7 menor atividade (40 U/g/min) com um mês, conforme demonstra a figura 3. Ferreira (2009) determinou a atividade da polifenoloxidase em polpas de cupuaçu congeladas, procedentes do estado de Roraima e município de Itacoatiara- AM tendo constatado que após 4 meses de estocagem em temperaturas de -25 a $-35{ }^{\circ} \mathrm{C}$, houve elevação da atividade da polifenoloxidase em $50 \%$ e $31 \%$, respectivamente, corroborando com os resultados de Lobo e Cano (1998) que verificaram incremento na atividade da polifenoloxidase de mamões congelados e armazenados em temperatura de $-24^{\circ} \mathrm{C}$ ao final de três meses. Segundo Pinheiro et al. (2009) a polifenoloxidase pode ser influenciada por fatores 
como tempo e temperatura de armazenamento, tendo encontrado valores de 328,37 e 369,54 U/g/min para a atividade de polifenoloxidase de abacates, armazenados a $0{ }^{\circ} \mathrm{C}$ e $10{ }^{\circ} \mathrm{C}$, respectivamente.
Relatou, ainda, que a polifenoloxidase apresentou comportamento oscilante durante os seis dias de armazenamento.

Figura 3. Atividade da polifenoloxidase nos genótipos de cupuaçu armazenados sob congelamento.

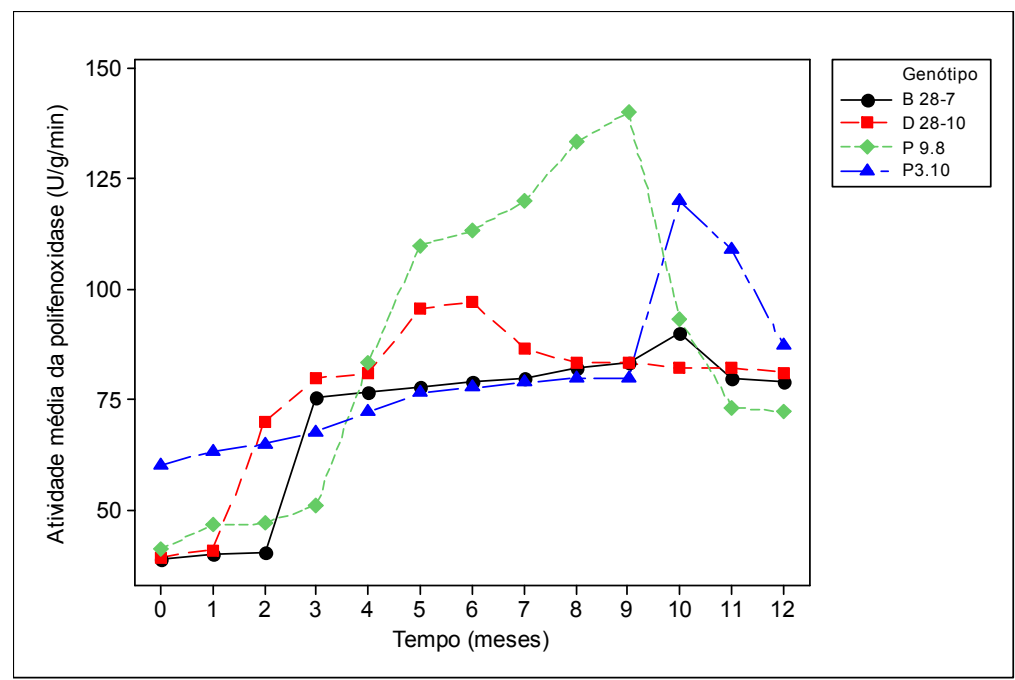

Fonte: Elaboração dos autores.

\section{Conclusões}

$\mathrm{O}$ armazenamento a $-30{ }^{\circ} \mathrm{C}$, durante um ano, das polpas dos quatro genótipos de cupuaçu não inibiu a atividade das enzimas polifenoloxidase e peroxidases. A polifenoloxidase apresentou aumento na sua atividade em função do tempo de estocagem sob congelamento com picos no sexto, nono e décimo mês e as peroxidases solúvel e insolúvel apresentaram oscilações na atividade enzimática nesse período.

Os resultados das análises físico-químicas das polpas dos quatro genótipos de cupuaçu apresentaram variações durante os doze meses de armazenamento sob congelamento, porém dentro dos padrões exigidos pela legislação vigente, com exceção do genótipo P 3-10 que apresentou acidez inferior.

\section{Agradecimentos}

À Embrapa Amazônia Ocidental pelo fornecimento das polpas de cupuaçu e à Coordenação de Aperfeiçoamento de Pessoal de Nível Superior (CAPES) pela concessão da bolsa de estudo.

\section{Referências}

AQUINO, A. C. M. S.; MOÉS, R. S.; CASTRO, A. A. Estabilidade de ácido ascórbico, carotenoides e antocianinas de frutos de acerola congelados por métodos criogênicos. Brazilian Journal Food and Technology, Campinas, v. 14, n. 2, p. 154-163, 2011.

ARAÚJO, L. M. Produção de alimentos funcionais formulados com xilitol a partir de cupuaçu (Theobroma grandiflorum) e maracujá (Passiflora edulis $f$. flavicarpa). 2007. Tese (Doutorado em Biotecnologia) Universidade Federal do Amazonas, Manaus. 
BERBICZ, F.; CLEMENTE, E. Avaliação da termoestabilidade e da regeneração da atividade da peroxidase extraída de laranja (Citrus spp.). Acta Scientiarum. Agronomy, Maringá, v. 23, n. 5, p. 1239$1242,2001$.

BRASIL. Ministério da Agricultura, Pecuária e Abastecimento. Instrução Normativa $\mathrm{n}^{\circ}$ 01, de 7 de janeiro de 2000. Aprova o regulamento técnico geral para fixação dos padrões de identidade e qualidade para polpa de frutas. Diário Oficial [da] União, Brasília, DF., 10 jan. 2000. Seção 1, p. 54-58.

BRUNINI, M. A.; OLIVEIRA, A. L.; VARANDA, D. B. Avaliação da qualidade de polpa de goiaba "paluma" armazenada a $-20^{\circ} \mathrm{C}$. Revista Brasileira de Fruticultura, Cruz das Almas, v. 25, n. 3, p. 394-396, 2003.

CANO, P.; MARIN, M. A.; FÚSTER, C. Freenzing of banana slices. Influence of maturity level and thermal treatment prior to freezing. Journal of Food Science, Chicago, v. 55, n. 4, p. 1070-1072, 1990.

CHITARRA, M. I. F.; CHITARRA, A. B. Pós-colheita de frutas e hortaliças: fisiologia e manuseio. Lavras: UFLA, 2005.

COHEN, K. O.; JACKIX, M. N. H. Estudo do liquor de cupuaçu. Ciência e Tecnologia de Alimentos, Campinas, v. 25, n. 1, p. 182-190, 2005.

COSTA, M. C. Conservação da polpa de cupuaçu (Theobroma grandiforum) por métodos combinados com emprego da tecnologia de obstáculos. 2002. Dissertação (Mestrado em Tecnologia de Alimentos) - Centro de Ciências Agrárias. Universidade Estadual do Ceará, Fortaleza.

DAIUTO, E. R.; VIEITES, R. L. Atividade da peroxidase e polifenoloxidase em abacate da variedade Hass, submetidos ao tratamento térmico. Revista Iberoamericana de Tecnología Postcosecha, México, v. 9, n. 2, p.106-112, 2008.

EVANGELISTA, R. M.; VIEITES, R. L. Avaliação da qualidade de polpa de goiaba congelada, comercializada na cidade de São Paulo. Segurança Alimentar $e$ Nutricional, Campinas, v. 13, n. 2, p. 76-81, 2006.

FARAONI, A. S. Efeito do tratamento térmico, do congelamento e da embalagem sobre o armazenamento da polpa de manga orgânica (Mangifera indica L.) $c v$ "ubá". 2006. Dissertação (Mestrado em Ciência e Tecnologia de Alimentos) - Universidade Federal de Viçosa, Viçosa.
FERREIRA, C. Q. Efeito do congelamento e da estocagem na atividade enzimática da polifenoloxidase (PFO) e peroxidase (POD) e na composição físicoquímica de polpa congelada de cupuaçu (Theobroma grandiflorum Schum). 2009. Dissertação (Mestrado em Ciência de Alimentos) - Universidade Federal do Amazonas, Manaus.

FREIRE, M. T. A.; PETRUS, R. R.; FREIRE, C. M. A.; OLIVEIRA, C. A. F.; FELIPE, A. M. P. F.; GATTI, J. B. Caracterização físico-química, microbiológica e sensorial de polpa de cupuaçu congelada (Theobroma grandiflorum Schum). Brazilian Journal Food Technology, Campinas, v. 12, n. 1, p. 9-16, 2009.

FREITAS, A. A.; FRANCELIN, M. F.; HIRATA, G. F.; CLEMENTE, E.; SCHMIDT, F. L. Atividades das enzimas peroxidase (POD) e polifenoloxidase (PPO) nas uvas das cultivares benitaka e rubi e em seus sucos e geleias. Ciência e Tecnologia de Alimentos, Campinas, v. 28, n. 1, p. 172-177, 2008.

HOLSCHUH, H. J. Isolamento, purificação e caracterização bioquímica da peroxidase de carambola (Averrhoa carambola, L.). 2000. Tese (Doutorado em Ciência de Alimentos) - Faculdade de Engenharia de Alimentos. Universidade Estadual de Campinas, Campinas.

INSTITUTO ADOLFO LUTZ - IAL. Normas analíticas do Instittuo Adolfo Lutz. Métodos químicos e físicos para análise de alimentos. São Paulo, 2008.

KHAN, A. A.; ROBINSON, D. S. Hidrogen donor specificity of mango isoperoxidase. Food Chemistry, London, v. 49, n. 4, p. 407-410, 1994.

LOBO, M. G.; CANO, M.P.Preservation of hermaphrodite and female papaya fruits (Carica papaya L., Cv Sunrise, Solo group) by freezing: physical, physicochemical and sensorial aspects. ZLebensm Unters Forsch A., Berlim, v. 206, n. 5, p. 343-349, 1998.

LOPES, A. S.; MATTIETTO, R. A.; MENEZES, H. C. Estabilidade da polpa de pitanga sob congelamento. Ciência e Tecnologia de Alimentos, Campinas, v. 25, n. 3, p. 553-559, 2005.

MANTOVAni, C.; ClEMENTE, E. Peroxidase and polyphenoloxidase activity in tomato in natura and tomato purée. Acta Scientiarum: Technology, v. 32, n. 1, p. 91-97, 2010.

MARTINS, V. B. Perfil sensorial de suco tropical de cupuaçu (Theobroma grandiflorum Schum) com valor calórico reduzido. 2008. Tese (Doutorado em Alimentos e Nutrição) - Faculdade de Engenharia de Alimentos. Universidade Estadual de Campinas, Campinas. 
MULYAWANTI, I.; DEWANDARI, K. T.; YULIANINGSIH. Effects of freezing and storage periods on characteristics of frozen sliced Arumanis mango. Indonesian Journal of Agriculture, Indonesia, v. 3, n. 1, p. 32-38, 2010.

NELSON, N. A fotometric adaptation of Somogyi method for the determination of glucose. The Journal of Biological Chemistry, Maryland, v. 153, p. 375-380, 1944.

NEVES, V. A. Ionically bound peroxidase from peach fruit. Brazilian Archives of Biology and Technology, Curitiba, v. 45, n. 1, p. 7-16, 2002.

NOGUEIRA, R. J. M. C.; MORAES, J. A. P. V.; BURITY, H. A.; SILVA JUNIOR, J. F. Efeito do estádio de maturação dos frutos nas características físicoquímicas de acerola. Pesquisa Agropecuária Brasileira, Brasília, v. 37, n. 4, p. 463-470, 2002.

NUNES, E. E.; VILAS BOAS, E. V.; PICCOLI, R. H.; XISTO, A. L. R. P.; VILAS BOAS, B. M. Efeito de diferentes temperaturas na qualidade de mandioquinhasalsa minimamente processada. Horticultura Brasileira, Brasília, v. 28, n. 3, p. 311-315, 2010.

OKTAY, M.; KUFREVIDGLU, I.; KOCACALISKAN, L.; SAKIROGLU, H. Polyphenoloxidase from Amasya Apple. Journal of Food Science, Chicago, v. 60, n. 3, p. 494-496, 1995.

OLIVEIRA, L. P. Seleção e aproveitamento biotecnológico de frutos encontrados na Amazônia para elaboração de bebida alcoólica fermentada utilizando levedura imobilizada. 2006. Tese (Doutorado em Biotecnologia) - Universidade Federal do Amazonas, Manaus.

PINHEIRO, A. C. M.; VILAS BOAS, E. V. B.; SILVA, L. C.; AlveS, A. P.; SElVA, M.; ChITARRA, A. B. Quality of fresh-cut avocado (Persea Americana Mill.) stored under different temperatures. Ciência e Agrotecnologia, Lavras, v. 33, n. 4, p. 1095-1102, 2009.
PORTE, A.; REZENDE, C. M.; ANTUNES, O. A. C.; MAIA, L. H. Redução de aminoácidos em polpas de bacuri (Platonia insignis Mart), cupuaçu (Theobroma grandiflorum Wild ex-Spreng Schum) e murici (Byrsonima crassifólia L.) processado (aquecido e alcalinizado). Acta Amazônica, Manaus, v. 40, n. 3, p. 573-578, 2010.

RANGANNA, S. Analysis and quality control for fruit and vegetable products. New Delhi: Tata McGraw-Hill, 1986.

SAHARI, M. A.; MOHSEN, B. F.; ZOHREH, H. E. Effect of low temperature on the ascorbic acid content and quality characteristics of frozen strawberry. Journal of Food Chemistry, Philadelphia, v. 86, n. 3, p. 357-363, 2004.

SANTOS, G. M.; MAIA, G.; SOUSA, P. H. M.; FIGUEIREDO, R. W.; COSTA, J. M. C.; FONSECA, A. V. V. Atividade antioxidante e correlações com componentes bioativos de produtos comerciais de cupuaçu. Ciência Rural, Santa Maria, v. 40, n. 7, p. 16361642, 2010.

SILVA, V. K. L.; FIGUEIREDO, R. W.; BRITO, E. S.; MAIA, G. A.; SOUSA, P. H. M.; FIGUEIREDO, E. A. T. Estabilidade da polpa do bacuri (Platonia insignis Mart.) congelada por 12 meses. Ciência e Agrotecnologia, Lavras, v. 34, n. 5, p. 1293-1300, 2010.

SOMOGYI, M. A. A new reagent for determination of sugars. The Journal of Biological Chemistry, Maryland, v. 160, p. 61-68, 1945.

YAMASHITA, F.; BENASSI, M. T.; TONZAR, A. C.; MORIYA, S.; FERNANDES, J. G. Produtos de acerola: estudo da estabilidade de vitamina C. Ciência e Tecnologia de Alimentos, Campinas, v. 23, n. 1, p. 92-94, 2003. 
